\title{
Untersuchungen zur Gattung und Metrik der Dionysos-Dithyrambe "Letzter Wille" von Fr. Nietzsche
}

[Exploring the genre and metrics of Nietzsche's Dionysus-Dithyramb "Letzter Wille"]

http://dx.doi.org/10.11606/1982-88372545136

Danilo Serpa ${ }^{1}$

\begin{abstract}
This paper analyses Nietzsche's Dionysus-Dithyramb "Letzter Wille" with regard to characteristics of the genre "hymn", of which the dithyramb can be considered a subgenre. The analysis considers two main concepts: gestures of speech and Freie Rhythmen (free rhythms). Gestures of speech as found in hymns could not be identified in the poem. Instead, the paper proposes that the attribution Freie Rhythmen (free rhythms) serves to characterize the poem. The metrical analysis is based on conceptualizations of Moennighoff and Schulz (2007) and Bunia (2014) and discusses features attributed to the genre "hymn". "Letzter Wille" is also observed in the context of Nietzsche's other poems. Finally, the motifs of the Dionysian and of self-reflection in Nietzsche are briefly examined.
\end{abstract}

Keywords: Dionysus-Dithyrambs; Free Rhythms (Freie Rhythmen); Hymn.

Zusammenfassung: In diesem Aufsatz wird Nietzsches Dionysos-Dithyrambe „Letzter Wille“ im Hinblick auf Charakteristiken der Gattung „Hymne“, welcher der Dithyrambus als Untergattung zugerechnet werden kann, analysiert. Zwei Aspekte werden besonders berücksichtigt: Sprachgebärden und Freie Rhythmen. Sprachgebärden der Hymnen konnten hier nicht ermittelt werden. Die metrische Struktur des Gedichts wird im Kontext des lyrischen Werkes Nietzsches besonders nach Begriffen von Moennighoff und Schulz (2007) sowie Bunia (2014) untersucht und auf andere, der Gattung „Hymne“ zugeschriebene Elemente bezogen. Dabei werden kompositorische Prozesse von „Letzter Wille“, wie die Spiegelung von seinen Teilen, herausgehoben, und seine Beziehung zu anderen Gedichten des Autors kurz betrachtet. Zum Schluss widmet sich die Analyse dem Motiv des Dionysischen bei Nietzsche.

Stichwörter: Dionysos-Dithyramben; Freie Rhythmen; Hymne.

\section{Einführung und Ziele des Textes}

Prinzipiell kann der Dithyrambus, der Gedichte der Antike bezeichnet, die dem Gott Dionysos gewidmet sind, als eine Untergattung der Hymne aufgefasst werden (generell

\footnotetext{
${ }^{1}$ Universidade de São Paulo, Faculdade de Filosofia, Letras e Ciências Humanas, Av. Luciano Gualberto, 403, Butantã, São Paulo, SP, 05508-010, Brasil. E-mail: daniloserpa@uol.com.br. ORCID: 0000-00023007-8904
}

(cc) BY-NC 
SERPA, D. - Untersuchungen der Dionysos-Dithyrambe "Letzter Wille"

zum Thema Bremer; Furley 2001: bes. 10-11; s. auch BuRdorf 2004: 300; 307). Im Folgenden wird der Versuch unternommen, Nietzsches Dionysos-Dithyrambe „Letzter Wille“ im Hinblick auf Charakteristiken der Gattung „Hymne“ zu untersuchen, vor allem wie sie sich in der deutschen Lyrik des 18. Jh.s etablieren; als Paradigmen der deutschen Hymne werden fünf Gedichte Klopstocks angesehen, die zwischen 1758-1760 in der Zeitschrift Der nordische Aufseher erscheinen, von denen „Die Frühlingsfeyer“ sicherlich das bekannteste ist; einige Sturm-und-Drang-Gedichte Goethes wie „Wandrers Sturmlied“, „Prometheus“ und „Harzreise im Winter“; Hölderlins Gedichte, die ungefähr zwischen 1799 und 1806 geschrieben wurden, wie „Die Wanderung“, „Der Rhein“ und „Andenken“ (BURDORF 2004: 302f.).

Die Untersuchung gilt hier den Sprachgebärden der Hymne und den Freien Rhythmen, welche als Merkmal des Genres in der deutschsprachigen Literatur herausragen. Es wird davon ausgegangen, dass Charakteristiken dieser Poesie, der deutschen Hymne, in Details noch auszuloten sind (BURDORF 2004; OESTERSANDFORT 2007). In einem weiteren Schritt werden anhand der Auseinandersetzung mit diesem Gedicht Nietzsches („Letzter Wille“) generelle Aspekte der Dionysos-Dithyramben in Betracht gezogen und kurz die Frage nach dem Dionysischen in dem Zyklus aufgeworfen.

\section{2. Über die Auswahl des Gedichts "Letzter Wille"}

Das Gedicht greift einen schon 1883 entstandenen Text wieder auf (BRUSOTTI 2011: 136; NK 6/2: 642). Obwohl ihn Nietzsche 1888 zusammen mit anderen der künftigen Dionysos-Dithyramben (DD) bearbeitet (GRODDECK 1991b: 453-459), gilt seine Vorlage als einer der frühersten Teile, der zum Zyklus gehören wird: Insgesamt umfassen die DD neun Gedichte und ihre Druckfassung stellt Nietzsche 1888/1889 fertig (NK 6/2: 641). In gewisser Hinsicht fällt „Letzter Wille“ aus dem Entstehungszusammenhang der anderen Gedichte des Zyklus sowie aus Konstellationen heraus, die diese unter sich formieren: 3 Gedichte („Nur Narr! Nur Dichter!“, „Unter Töchtern der Wüste“ und „Klage der Ariadne") sind aus dem vierten Teil des Zarathustra entnommen, der 1885 im Privatdruck erscheint (KSA 4: 7), während „Letzter Wille“ im Zusammenhang mit dem dritten Teil des Zarathustra steht, mit dem gestrichenen Kapitel-Entwurf „Von einem Sieg“(GRODDECK 1991a: XLIII-XLIV); die anderen 5 Gedichte fasst Nietzsche ab 1888 grundsätzlich aus auch unveröffentlichten dichterischen Entwürfen ab (BRUSOTTI 2011: 
SERPA, D. - Untersuchungen der Dionysos-Dithyrambe "Letzter Wille"

136, NK 6/2: 642). Es ist dann naheliegend, dass „Letzter Wille“ innerhalb des Zyklus Besonderheiten aufweist, auch was ihre formellen Eigenschaften angeht. Es spricht ferner für eine Analyse dieses Gedichts, dass ihm im Vergleich zu anderen DD noch wenig Beachtung geschenkt wurde.

\section{3. "Letzter Wille" und Charakteristiken der Gattung}

Festzustellen ist, dass „Letzter Wille“ Sprachgebärden, die typisch für die deutsche Hymne sind und die Gattung auch in der Antike kennzeichnen, nicht enthält. Gemeint sind damit vor allem erstens eine Anrufung, die Hinwendung an eine Entität, zweitens eine preisende Beschreibung bzw. Erzählung von deren Machtbereich, Eigenschaften, und/ oder Taten und drittens eine sich daran anschließende Bitte (SERPA 2014). Gewiss stellt „Letzter Wille“ eine Art Huldigung des „Freund[es]“ (KSA 6: 388, v. 3) ${ }^{2}$ dar, aber sie ist keinesfalls kletisch, ${ }^{3}$ wendet sich nicht direkt an ihn und fällt in diesem Sinn ganz aus dem Zusammenhang der Hymne heraus. Zu „Letzter Wille“ wurde gerade eine Stelle des Abschnitts „Von einem Sieg“ verworfen, in dem zumindest einige der oben den Hymnen zugeschriebenen Sprachgebärden zu ermitteln sind: „Oh du mein Wille, mein In-Mir, Über-mir! du meine Nothwendigkeit! Gieb, daß ich also siege — und spare mich auf zu diesem Einen Siege!“ (eKGWB: NF-1883, 20[11]). ${ }^{4}$ In der Konjunktur W. Groddecks (1991b: 456) waren diese Zeilen „Von einem Sieg““ „eine Hymne oder ein ,Gebet', das nach dem Tod Zarathustras gesprochen wird“. Dem Gedicht werden aber solche hymnischen Züge nicht verliehen.

Das Gedicht präsentiert auch nicht die Charakteristiken der Poesie, die im 18 Jh. Gegenstand einer Nachahmungsproblematik für bedeutende Autoren der deutschen Literatur wird: Die Dithyrambe. Wie dem Gedicht Klopstocks (2006: 18-38) „Auf meine Freunde“ (1747) und dem Text Herders „Pindar und der Dithyrambensänger“ (1767), den Nietzsche gekannt haben soll, ${ }^{5} \mathrm{zu}$ entnehmen ist, orientiert sich da die Idee einer

\footnotetext{
${ }^{2}$ Das Gedicht wird nach KSA 6: 388 zitiert, aber anders als in der KSA ist hier der Titel nicht als erste Zeile gezählt.

${ }^{3}$ Das Gedicht mündet in keine Epiphanie. Zu dieser Charakteristik in Hymnen s. z. B. Bremer; Furley (2001: 61).

${ }^{4}$ http://www.nietzschesource.org/\#eKGWB/NF-1883,20.

${ }^{5}$ In den Worten A. U. Sommers: „N. kannte die als Sammlung von Fragmenten deklarierte Schrift Herders Über die neuere deutsche Litteratur. Die erste dieser ,Sammlungen' (1767) enthält ein Kapitel über ,Pindar und die Dithyrambensänger““ (NK 6/2: 647).
} 
SERPA, D. - Untersuchungen der Dionysos-Dithyrambe "Letzter Wille"

dithyrambischen Poesie stark an der horaz'schen Ode IV , 2. ${ }^{6}$ Die Bildung neuer Wörter, ,kühne Zusammensetzungen“ (HERDER 1767: 308), ${ }^{7}$ die als ein Merkmal der Dithyrambe betrachtet und in der hymnischen Dichtung dieser Zeit (man denke etwa an Goethes sogenannte Frankfurter Hymnen, wie „Wandrers Sturmlied“8 oder „Prometheus“) ergiebig praktiziert wurden, sind in „Letzter Wille“ mit der Ausnahme vielleicht von „Vordenklich“ (KSA 6: 388, V. 10; s. GRODDECK 1991b: 106) nicht zu finden. Das unterscheidet es auch von anderen Gedichten der DD, in denen sich ,kühne Zusammensetzungen“ bemerkbar machen (z. B.: „Lammsmilch-Wohlwollen“, KSA 6: 379, V. 69; „Herzens-Kohlenbecken“, KSA 6: 398, V. 3; „Allerwelts-Blechklingklang“, KSA 6: 403, V. 34). ${ }^{9}$ In einem Vergleich zu anderen Gedichten dieses Zyklus von Nietzsche zeigt es einen gehaltenen Ton.

Im Text „Pindar und der Dithyrambensänger“ preist Herder (1769: 330s.) bei seinem Modell von einer enthusiastischen Dichtung, bei Pindar, der auch einen Kontrast zu dem „Dithyrambensänger“ des Titels bildet, die Unordnung, die Schwünge und Sprünge seiner Gesänge. Diese Elemente manifestieren sich in syntaktischen Brüchen, Auslassungen, raschen Wechseln von Themen, kühnen Enjambements in den von solch einem Pindar-Bild inspirierten Dichtungen Goethes und prägen auch im gewissen $\mathrm{Ma}$ den Stil von einigen Dionysos-Dithyramben Nietzsches, erscheinen aber - in diesem Vergleich - auf eine sehr gemilderte Weise im Gedicht „Letzter Wille“. Von Unordnung kann hier kaum die Rede sein. Zwar gibt es Auslassungen von Konnektoren, ist nur

\footnotetext{
${ }^{6}$ Diese Ode (HoRACE 1995: 286) stellt einen Zusammenhang zwischen dem Dithyrambus (V. 10) und der Dichtung Pindars her, welche „,neue Wörter“ erfindet (,nova [...]/ verba devolvit“) (VV. 10-11) und ,von festen Silbenmaßen gelöst“ ist („,numerisque fertur/ lege solutis“) (VV. 11-12). Unter anderem ist die Strommetapher für die poetisch-schöpferischen Kräfte, die in diesem Gedicht Horazens herrscht, sehr produktiv in der deutschen Dichtung der Zeit. Zur Rezeption und zum Verständnis dieser Ode in zweiter Hälfte des 18. Jh.s. (VÖHLER 2005: 152-155). Mit der Ode IV, 2 wird ein „Modell der recusatio“ (id. ibid.) verbunden, eine Warnung vor den Gefahren einer aemulatio Pindars und der seiner Dichtung attribuierten Eigenschaften. Zugleich ordnet sie sich in den Rahmen eines Lobs und einer Wertschätzung Pindars ein und dient auch als Anregung dazu, dem Dithyrambus zugeschriebene Elemente in die deutsche Dichtung der Zeit (um die zweite Hälfte des 18. Jh.s) zu integrieren. Diese Ambivalenz lässt sich am zitierten Gedicht Klopstocks erkennen, das einerseits Pindar preist (KLOPSTOCK 2006: 18, VV. 5-6), eingangs ankündigt, die Freunde „in mächtigen Dithyramben“ (V. 4) zu singen und zu feiern, andererseits aber selbst von der Alternative der pindarischen Dichtung (VV. 5-8) abweicht, indem es ,zu Strophen“ (V. 5) wird - und zwar zu einer alkäischen Ode.

${ }^{7}$ In: https://www.uni-due.de/lyriktheorie/texte/1767_2herder.html\#edition.

${ }^{8}$ Dieses Gedicht hat Goethe selbst als „Dithyrambe“ bezeichnet, im 12. Buch des dritten Teiles (1814) seiner poetischen Autobiographie Dichtung und Wahrheit (GOETHE 1975: 579)

${ }^{9}$ Andreas Sommer (NK 6/2: 646) versteht die folgende Passage der Poetik Aristoteles' (1459a8f.) als einen Hinweis darauf, die Bildung neuer Komposita kennzeichne die Sprache des Dithyrambus: „Von den Nomina sind die doppelten am meisten dem Dithyrambos angemessen“. Man sieht an den oben gegebenen Beispielen, dass Nietzsche nach dem Schema eines durch Strich in zwei Teile gegliederten Worts mehrere Wörter aneinanderreiht.
} 
SERPA, D. - Untersuchungen der Dionysos-Dithyrambe "Letzter Wille"

einmal ein flektiertes Vollverb in einem Hauptsatz (V. 15, KSA 6: 388) zu finden, leiten viele Gedankenstriche nachtragende Erweiterungen ein, aber sie scheinen zu keinem übermäßig entfesselten Pathos zu führen und eher Zeichen einer noch in Grenzen sich haltenden Begeisterung zu sein. Die echoartigen Wiederholungen und Verdopplungen führen hier zu keinen „quasi-stabreimenden Alliterationen“ (NK 6/2: 656), die anderen Passagen des Zyklus kennzeichnen. ${ }^{10}$

\title{
4. Freie Rhythmen
}

Letzter Wille.

\author{
So sterben, \\ wie ich ihn einst sterben sah -, \\ den Freund, der Blitze und Blicke \\ göttlich in meine dunkle Jugend warf. \\ $5 \quad$ Mutwillig und tief, \\ in der Schlacht ein Tänzer -, \\ unter Kriegern der Heiterste, \\ unter Siegern der Schwerste, \\ auf seinem Schicksal ein Schicksal stehend, \\ hart, nachdenklich, vordenklich -: \\ erzitternd darob, dass er siegte, \\ jauchzend darüber, dass er sterbend siegte -: \\ befehlend, indem er starb \\ - und er befahl, dass man vernichte... \\ 15 So sterben, \\ wie ich ihn einst sterben sah: \\ siegend, vernichtend...
}

Das Gedicht hat keine strophische Ordnung ${ }^{11}$ und keine Reime. Seine Freien Rhythmen sind ein Merkmal, das es mit der deutschen Hymne des 18. Jh.s teilt. „Letzter Wille“ zeigt rhythmische Parallelismen - Parallelismen in der Verteilung von Hebungen und Senkungen - mit Variationen, wie zum Beispiel zwischen den Versen 6-8. ${ }^{12}$ Die Verse weisen aber kein durchgehendes Versmaß auf. Es gibt keine gleiche Anzahl von

\footnotetext{
${ }^{10}$ A. Sommer (NK 6/2: 655s.) führt als Beispiel die Verse 53-67 des ersten Gedichts des Zyklus an: „Oder dem Adler gleich, der lange,/ lange starr in Abgründe blickt,/ in seine Abgründe.../ — oh wie sie sich hier hinab,/ hinunter, hinein,/ in immer tiefere Tiefen ringeln! — Dann, plötzlich,/ geraden Flugs/ gezückten Zugs/ auf Lämmer stossen/ jach hinab, heisshungrig,/ nach Lämmern lüstern, gram allen Lamms-Seelen,/ grimmig gram".

${ }^{11}$ In dem Sinn von Strophen, die "gleichmäßig gebaut sind" (BURDORF 2015: 98).

${ }^{12} \mathrm{Zu}$,phonological and rhythmic correspondences between lines“ als Charakteristik der Freien Rhythmen (s. BOWERS; CHISHOLM 2012: 525).
} 
SERPA, D. - Untersuchungen der Dionysos-Dithyrambe "Letzter Wille"

Hebungen pro Vers. Keine feste Regelmäßigkeit ergibt sich aus der Abfolge von Hebungen und Senkungen.

Was die Metrik betrifft, ist ein Element, das besonders auffällt und an der rhythmischen Gliederung des Gedichts teilnimmt, der Amphibrachys, eine dreisilbige Einheit, die in der deutschen Metrik eine Sequenz von Senkung, Hebung und Senkung bezeichnet. Er erscheint schon in der ersten Zeile („So sterben“) und an bedeutenden Stellen und Wendungen des Gedichts, wie am Ende von Versen (,,vernichte“), in Wörtern, die den „Freund“ bezeichnen („ein Tänzer“, „der Schwerste“), in partizipialen Formen, die das Gedicht prägen (,,erzitternd“, ,,befehlend“), und am Ende (,,vernichtend“), wobei „Siegend, vernichtend“ (-uu-u), der letzte Vers, mit jener metrischen Klausel ausklingt, die für die hexametrische Dichtung und besonders für Oden der Antike sowie für viele Gedichte der deutschen Hymne charakteristisch ist, mit dem Adonius.

Die Freien Rhythmen nehmen an den vielen Spiegelungen teil, die das Gedicht bietet. Auch in dieser Hinsicht beziehen sich z. B. „erzitternd darob“ (u-u u-) und ,jauchzend darüber“ (-u u-u) in den Versen 11 und 12 chiastisch aufeinander. Der Vers 9 bildet die Mitte des Gedichts, eine Achse. Das Polyptoton teilt den Vers in zwei Hälften mit gleicher metrischer Beschaffenheit (,,auf seinem Schicksal | ein Schicksal stehend“) ( $\mathrm{u}-\mathrm{u}-\mathrm{u} \mid \mathrm{u}-\mathrm{u}-\mathrm{u})$. In der Ringkomposition des Gedichts spiegelt das Ende den Anfang wider. Dieses Strukturmerkmal passt zu der Thematisierung des Verhältnisses des „Ich“ zu dem „Freund“, seines Reflektierens, wobei selbst der Augenkontakt eine wichtige Rolle spielt: Das Ich „sah“ den Freund (KSA 6: 388, V. 2), der ,Blicke in seine dunkle Jugend geworfen hatte' (VV. 3-4).

Aus der kurzen Analyse oben sollte sich ergeben, dass die Bestimmung der Freien Rhythmen als „Verse ohne Reime, ohne Strophenordnung und ohne durchgehendes Versmaß“, die „Anleihen bei antiken Versformen machen“ (SCHULZ; WERNER 2007: 253), auf das Gedicht zutrifft. Mögen diese „Anleihen bei antiken Versformen“ (id. ibid.) nicht so fassbar sein wie z. B. bei Hölderlin, dessen Gedicht „Wie wenn am Feiertage“ „noch deutlich das Vorbild, den Ausgangspunkt des alkäischen Odenverses verraten (SzONDI 1975: 202): ${ }^{13}$ „Letzter Wille“ - ein Gedicht ohne durchgehendes Versmaß bedient sich Strukturen der antiken Metrik bzw. der klassischen deutschen Literatur. Ein Beispiel ist die Adonius-Klausel („Siegend, vernichtend“, KSA 6: 388, V. 17). Auffallend ist außerdem der Amphibrachys, der an den vielen Wiederholungen und spiegelbildlichen

\footnotetext{
${ }^{13}$ Wagenknecht (1993: 94s.) führt Beispiele dieser Art in den frei-rhythmischen Hymnen Klopstocks an.
} 
SERPA, D. - Untersuchungen der Dionysos-Dithyrambe "Letzter Wille"

Beziehungen zwischen Teilen des Gedichts mitwirkt. Aber ein bestimmtes metrisches Vorbild als Ausgangspunkt des Gedichts ist schwer feststellbar.

Der antike Versfuß Amphibrachys ist übrigens ,nicht als selbstständiges Silbenmaß belegt“ (STEINHAGEN 2007: 19). In der deutschen Literatur finden sich jedoch spärlich Gedichte, die fast ausschließlich aus diesem Versfuß bestehen (id. ibid.), wie das sogenannte „Lied der Parzen“ in Goethes Iphigenie auf Tauris (1787). ${ }^{14}$ Auch im „Hyperions Schicksalslied“ (1799), Gedicht mit „freirhythmischen Versen“ (J. Schmidt, SW I: 620), ist im letzten Abschnitt der Amphibrachys unüberhörbar (,Doch uns ist gegeben“; „es schwinden, es fallen“/ „Die leidenden Menschen“; „Wie Wasser von Klippe/ Zur Klippe geworfen“, SW I: 207, V. 16; 18-19; 22-23). Im Fall von „Letzter Wille“ ist eine Sequenz von zwei Amphibrachen wiederum selten. Es gibt sie im dritten Vers: „der Blitze und Blicke“ (KSA 6: 388). Dieser Versfuß lässt sich da jedoch eher isoliert finden, wie die oben angeführten Beispiele zeigen. Was die Motive dieser Gedichte angeht, bietet sich auch ein Vergleich an (ohne direkte bzw. bewusste Bezüge und Wiederaufnahmen bestimmen zu wollen). Sie bewegen sich in einer griechischmythologischen Vorstellungwelt (,Letzter Wille“ steht in diesem Rahmen durch den Titel des Zyklus) und thematisieren das Schicksal. Während aber das „Lied der Parzen“ und „Hyperions Schicksalslied“ in ihrer Schilderung des menschlichen Lebens nach einer tragischen Gesinnung dessen Aufblühen und Verwelken, Erhöhung und Fall im Unterschied $\mathrm{zu}$ den Göttern behandeln, werden menschliche und göttliche Züge in „Letzter Wille“ angenähert, indem das Vorbild des Ich, dessen Tod den Charakter eines Opfertodes bekommt, das Attribut ,göttlich“ (KSA 6: 388, V. 4) trägt.

Anders als in den Dionysos-Dithyramben dominiert in der publizierten Versdichtung Nietzsches - Gedichte aus Zarathustra ausgenommen, aus dem Texte der Dionysos-Dithyramben stammen - die Realisierung von regelmäßigeren Formen der Lyrik. In den Idyllen aus Messina (,1882 erschienen in der Chemnitzer Internationalen Monatsschrift“", ZIEMANN 2011: 152) sind die Gedichte strophisch mit Reimen aufgebaut. Der in meist 3- oder 4-hebigen Versen jambische oder trochäische Rhythmus überwiegt, wobei es im Zyklus auch Gedichte wie „Lied des Ziegenhirten“ oder „Vogel Albatross“ gibt, die Verse mit verschiedener Hebungszahl alternieren lassen: im ersten Fall wechseln sich 3- und 2-hebige mit freier Füllung ab; im zweiten genannten Gedicht 3- und 5-hebige

\footnotetext{
${ }^{14}$ Fast jeder Vers dieses ,Liedes‘ besteht aus zwei Amphibrachen. Der erste Abschnitt lautet: "Es fürchte die Götter/ Das Menschengeschlecht!/ Sie halten die Herrschaft/ In ewigen Händen,/ Und können sie brauchen/ Wie's ihnen gefällt.” (GOETHE 2008: 605, VV. 1726-1766).
} 
SERPA, D. - Untersuchungen der Dionysos-Dithyrambe "Letzter Wille"

Jamben. Der Sinn Nietzsches für (Selbst)Ironie macht sich in dem letzten Gedicht des Zyklus (,Vogel-Urtheil“) stark bemerkbar, das eine Art Einweihungsszene des Dichters darstellt: Das Ich, das sich unter Bäumen zur Ruhe gesetzt hat, ärgert sich über ein von ihm gehörtes ,Ticken“, bis es endlich nachgibt: „[B]is ich gar, gleich einem Dichter/ Selber mit im Tiktak sprach.// Wie mir so im Versemachen/ Silb' um Silb' ihr Hopsa sprang/ Musst ich plötzlich lachen, lachen/ Eine Viertelstunde lang“ (KSA 3: 342, VV. 7-12). Nach Ziemann (2011: 153s.) weisen diese Verse schon auf eine Haltung „der wachen und reichen Passivität im Erleben der Zeit" hin, die Gedichte des Anhangs der Neuauflage der Fröhlichen Wissenschaft (1887) bestimmt (das bekannteste Gedicht in dieser Hinsicht ist wohl „Sils-Maria“). Sie sind immerhin ein Zeugnis des ambivalenten bzw. skeptischen Verhältnisses des Autors gegenüber solchermaßen rhythmisierten Versen und sogar der Dichtung selbst; ${ }^{15}$ Ambivalenz und Skepsis, die auch in Gedichten des Zyklus der Dionysos-Dithyramben deutlich zum Ausdruck kommen.

Ohne die Absicht, die Formen der Lyrik und ihr Vorkommen bei Nietzsche aufzulisten: In seinem veröffentlichten Werk ist eine Tendenz zur Bereicherung und Auflösung des regelmäßig metrischen Gefüges zu beobachten; oder, in der Begrifflichkeit Bunias (2014: bes. 40-49), eine Abwandlung von einem ,normativen“ zu einem „deduktiven Versprogramm“. D. h.: Während generell den Gedichten der Idyllen aus Messina oder der Fröhlichen Wissenschaft eine abstrakte metrische Formel, ,eine abstrakte Vorgabe im metrischen Raum“ (BUNIA 2014: 44) zugrunde liegt, die, aus der Tradition mehr oder weniger bekannt, auf den prosodischen Raum wirkt (natürlich kann die Realisierung eines jeden solcher Verse im Einzelfall diskutiert werden und jedes Gedicht innerhalb seiner metrischen Struktur ein sehr individuelles Gepräge haben), ist im Fall der Dionysos-Dithyramben auf kein metrisches Muster dieser Art zu schließen, das sich in jedem Vers (oder in jedem Vers einer bestimmten Gruppe von Versen) wiederholt und seine Gültigkeit hat. Selbstverständlich liegen hier keine Wertschätzung des einen „Programms“ gegenüber dem anderen und keine Erklärung über die Entstehung der Freien Rhythmen bei Nietzsche vor; es geht vielmehr um eine Beschreibung von seinen Versen, und zwar eine abstrakte, denn ab einem gewissen Punkt koexistieren sie. Es gibt, wie gesagt, die Gedichte in Zarathustra (aus dem vierten Teil z. B., KSA 4: 313-

\footnotetext{
${ }^{15}$ Diese Haltung Nietzsches der Poesie gegenüber zeigt sich zum Beispiel im 84. Abschnitt der im selben Jahr (1882) erschienen Fröhlichen Wissenschaft (KSA 3: 439-442).
} 
SERPA, D. - Untersuchungen der Dionysos-Dithyrambe "Letzter Wille"

317; 371-374). Diese Variation lässt sich aber in den Eckpunkten seines Werkes beobachten.

Im Verlauf der Versdichtung Nietzsches wird eine Art Aneignung neuer Formen erkennbar. In dem Zyklus „Scherz, List und Rache“, der in Die fröhliche Wissenschaft 1882 erscheint, sind neben Gedichten, in denen Hebung und Senkung (oder umgekehrt) alternieren, und Gedichten mit einer bestimmten Zahl von Hebungen pro Vers (üblicherweise 3- oder 4-hebig), neben Liedern (die z. B. einem 4-hebigen Vers einen 3hebigen folgen lassen) und Blankversen (wie das Gedicht „Interpretation“, KSA 3: 357) ein elegisches Distichon („An die Tugendsamen“) (KSA 3: 354) und das Gedicht „Für Tänzer“" (KSA 3: 356) auffallend, das in seinen 3 Versen keine Regelmäßigkeit aufweist, es sei denn, sie seien alternierend zu lesen. Der Reim (der Endreim) ist eine Komponente dieser Gedichte. Er zeigt sich auch an den Gedichten, die die „Lieder des Prinzen Vogelfrei“" ausmachen. Diese erscheinen 1887 als Anhang der Neuauflage der Fröhlichen Wissenschaft (KSA 3: 639-651). Wie der Titel schon verrät, erweisen sich viele Gedichte dieses Zyklus als eine Bearbeitung, in der das Silbenmaß weitgehend beibehalten wird, von Gedichten der Idyllen aus Messina (deren erstes Gedicht den „Prinz Vogelfrei““ vorstellt). Ein besonderes metrisches Gebilde stellt das Gedicht „Rimus remedium“ vor. Es hat fünf Strophen mit je sechs Versen; drei von denen sind 2-hebig und die anderen drei Verse 4-hebig, aber die Sequenz von 2- und 4-hebig ist nicht in jeder Strophe gleich, und der dritte Vers der ersten Strophe scheint eine Ausnahme zu bilden. Das Gedicht „An Goethe“, das den Zyklus eröffnet, ahmt deutlich den Chorus Mysticus, die Schlussverse von Faust II, nach. Charakteristisch für sie sind die 2-hebigen Verse im daktylischen Duktus.

Der Chorus Mysticus wird schon in den ersten Versen dieses Textes Nietzsches durch Repetition von Wörtern aufgerufen. Das Gedicht, das ,An Goethe“ gerichtet ist, stellt sich polemisch gegen ihn bzw. diese seine Verse. Im Kontext des Werks Nietzsches, im Zarathustra (II, KSA 4: 110; 164), verbindet sich das Zitat aus dem Chorus Mysticus mit der Provokation, die nicht selten bei dem Autor anzutreffen ist, dass die Dichter ,zuviel“ lügen. In „An Goethe“ werden diese Verse des Fausts aufgegriffen, um sich mit einem anderen Thema auseinanderzusetzen, das auch in den angeführten Passagen Zarathustras eine bedeutende Rolle spielt: Die Kritik Nietzsches an einer Abwertung, Schlechtmachung ,alles Vergänglichen“, an einer Idee des ,Unvergänglichen‘, einer göttlichen Sphäre, eines Jenseits, dem eine ,höhere‘ Bedeutung zugeschrieben werde als 
SERPA, D. - Untersuchungen der Dionysos-Dithyrambe "Letzter Wille"

der Erde, jeglichem Weltlichen. Schon eine Trennung zwischen Diesseits und Jenseits sei in Frage zu stellen, sowie der Impuls, sich anstatt der Erde dem Himmel (als Symbol dieser göttlichen Sphäre begriffen) zuzuwenden. Dem Ewig-Weiblichen des Chorus Mysticus, das den Weg nach oben bahnt und als ein Prinzip der Transzendierung verstanden werden kann, wird das „Ewig-Närrische“ (KSA 3: 639, V. 11) entgegengesetzt, das für die Mischung der Sphären, das Sich-Einmischen, sowie für die Abwendung von dem ,hinan“ des Chorus Mysticus (GoETHE 2007: 1064, V. 12.111) zum „Welt-Spiel“ (KSA 3: 639, V. 9) steht. Daran sieht man, dass die Figur des Narren bei Nietzsche hier auch positiv konnotiert ist. Ein anderes Beispiel dafür gibt H. Hiebel (2005: 63) bei der Analyse des Gedichts „Der Freigeist“ (1884, von Nietzsche unveröffentlicht): Der Narr stelle sich als, wise fool ${ }^{\star}$ heraus und sei eine Identifikationsfigur für das Ich. Im Zyklus „Lieder des Prinzen Vogelfrei“ können diese Züge in ihrer Ambivalenz im Gedicht „Narr in Verzweiflung“ (KSA 3: 646) gesehen werden. In einem Vergleich zwischen Narren und Weisen wird da in gewisser Hinsicht dem Narren der Vorzug gegeben.

Im Gedicht „Aus hohen Bergen“, das das Buch Jenseits von Gut und Böse (1886) abschließt, sind Motive und Vokabular den Dionysos-Dithyramben nah. Selbst die Länge ist mit vielen DD vergleichbar. Ein Zug des Erhabenen zeigt sich in der „Eis- und Felsenreich“-Landschaft (KSA 5: 242, V. 34) mit ihren ,Abgründen“(V. 13). Das Gedicht behandelt erneut ein Thema, das sich durch Nietzsches Lyrik durchzieht: die Einsamkeit. Zudem haben wir es hier mit einem gespalteten Ich zu tun, das ,sich selber fremd“, „ein Andrer“(V. 21) geworden ist. Die Darlegung seines (inneren) Konflikts bedient sich einer Kampfmetaphorik. Das Ich, das gegen sich selbst kämpft (V.23) und in Jäger und Beute (V. 35) gespalten ist, ähnelt dem, was in der Dionysos-Dithyrambe „Zwischen Raubvögeln“ geschildet wird. In diesem Aspekt zeigt „Aus hohen Bergen“ Parallelen auch zu „Letzter Wille“, in dem Siegen und Erliegen des Ich eine Einheit bilden. Ferner ist es auch von Belang, wenn man „Letzter Wille“ in diesen Kontext stellt, dass Zarathustra als der „Freund“ (KSA 5: 243, V. 68-69; 73) bezeichnet wird. Im Bereich der Metrik sind das Gedicht in Jenseits von Gut und Böse und die DD unterschiedlich. „Aus hohen Bergen“"gliedert sich in Strophen mit je 5 Versen, gereimt nach dem Schema abbaa. Es sind Blankverse, fünfhebiger Jambus, außer dem zweiten Vers jeder Strophe, der einen zwei-hebigen Jambus bildet. 
SERPA, D. - Untersuchungen der Dionysos-Dithyrambe "Letzter Wille"

Bereits im Rahmen der nachgelassenen Schriften gibt es das sogenannte VenedigGedicht (auch als „Gondellied“ bekannt) Nietzsches von 1888 mit seinen „freirhythmisch wirkenden Versen“ (GRODDECK 2011: 25; für das Datum, id. ibid. S. 20; da schreibt er: Es „hat keinen vom Autor stammenden Titel“). Es befindet sich in den nachgelassenen Schriften Ecce homo (1888/1889, KSA 6: 291) und Nietzsche contra Wagner (1888, KSA 6: 421). Groddecks (2011: 25) behutsame Formulierung über seine „freirhythmisch wirkenden Verse [...]“ erklärt sich aus der Aufbaustruktur dieses Gedichtes ohne Reim (ohne Reimschema). Versanfang und -ende sind immer betont. Die Verse haben entweder 3 oder 4 Hebungen, was zu seinem liedhaften Charakter beiträgt. Aber es ist nicht voraussehbar, wann ein Vers 3- oder 4-hebig ist; es ist nicht regelmäßig in diesem Aspekt. Das Gedicht hat zwei Abschnitte: Der erste mit sieben und der zweite mit fünf Versen. Die ersten vier Verse des ersten Abschnitts sind 3-hebig. Dann alternieren in den letzten drei Versen 4- und 3-Heber. Die ersten vier Verse des zweiten Abschnitts sind 4-hebig und er endet mit einem 3-hebigen. Insgesamt sind sechs Verse 3-hebig und sechs 4-hebig im Gedicht.

Die Einordnung der Verse der DD als Freie Rhythmen hat eine Tradition und ist in der Forschung etabliert (KoMMERELl 1956, MoENNIGHOFF; SCHUlz 2007: 253), wenn auch einige Vorbehalte in dieser Bestimmung zu spüren sind. Beispielsweise spricht $\mathrm{H}$. Hiebel (2005: 69) in Bezug auf die DD vom „Weg zum vers libre“, ,zum freien Vers“ es ist dennoch anzunehmen, dass Hiebel in dieser Formulierung die DD doch als frei rhythmische Gedichte versteht, sie aber in ihrer Nähe zu Tendenzen der Poesie des 20. Jh.s begreift.

Wenn man eine rein metrische Perspektive einnimmt, bereitet die Unterscheidung zwischen beiden Versarten (Freie Rhythmen und Freie Verse) viele Schwierigkeiten, selbst in Rücksicht auf die Definitionen, die Georg-Michael Schulz und Burkard Moennighoff (2007) vorschlagen. Demnach "greifen" die freien Verse "nicht auf antike Verselemente zurück" und stehen der Prosa näher (MoEnNighoff 2007: 253). Die ,Anleihen bei antiken Verselementen' (id. ibid.) sind aber von Fall zu Fall nicht leicht feststellbar. Außerdem ist ein prosaischer Charakter als Zug der freien Rhythmen schon im 18. Jh. erkennbar (s. zu diesem letzten Punkt KomMERELl 1956: 432-433, WerLE 2012: 488; 483). Zur prosaischen bzw. nicht-prosaischen Eigenschaft des Gedichts tragen dann andere Elemente bei, die sich nicht ausschließlich auf die rhythmische Struktur des Verses beschränken. Moennighoff und Schulz (2007: 253) weisen darauf hin, dass 
SERPA, D. - Untersuchungen der Dionysos-Dithyrambe "Letzter Wille"

„Gedichte in F.n Rh. [...] durch hohen, auch hymnischen Stil gekennzeichnet“" sind, während im Freien Vers ,der feierlich-gehobene Ton [...] vielfach aufgegeben [wird]“ (id. ibid.). ${ }^{16}$

$\mathrm{Zu}$ der Gehobenheit des Stils in freirhythmischen Gedichten des 18. Jh.s zählen z. B. Anredeformen wie o; superlativistische Sprache; syntaktische Inversionen, die als eine Folge haben, dass die Sprache antikisierend wirkt (auch das häufige Vorkommen von Partizip Präsens in prädikativer Stellung hat einen ähnlichen Effekt); Auslassungen von Konnektoren, die u. a. die Idee einer enthusiastischen sowie in der Not sich artikulierten Rede vermitteln. Wie oben angezeigt, weist „Letzter Wille“ in etwas bescheidenem Maß solche Elemente auf, aber als Teil eines Zyklus (der Dionysos-Dithyramben) steht es im engen Zusammenhang mit Gedichten, die diese Charakteristiken haben (obzwar ihr Gepräge anders ist als das der Hymnen des vorangehenden Jahrhunderts). Außerdem bedient es sich einer reichen Adjektivierung, die sich zu Superlativen (KSA 6: 388, VV. 7-8) zuspitzen. Sein agonistischer Charakter, der ein Todespathos nicht ganz verbirgt und in einer Art Todesapotheose gipfelt, spielt wie von ferne auf „die agonale Struktur des Erhabenen“ (Ausdruck von MENNINGHAus 1991: 12 in seiner Untersuchung zu Bestimmungen über das Erhabene) an. Es schildert eine Bewegung, die zwischen Auflösung und Erhaltung, Selbstbehauptung des Ich schillert. Auch die Todesthematik des Gedichtes „Letzter Wille“ ist Hymnen des 18. Jh. s. nicht fremd: in diesen finden sich oft Darstellungen von gefährlichen, das Ich bedrohenden und sogar vernichtenden Szenarien oder Vorstellungen des Todes bzw. Untergangs des Ich.

Ein Grund, den M. Kommerell (1956: bes. 431ff.) auch erwägt, um die DD in die Nähe der freirhythmischen Hymnen Klopstocks, Goethes und Hölderlins zu rücken, liegt in ihrer häufigen Behandlung des Verhältnisses des Menschen, des Dichters zum Göttlichen, wobei sich die Anrede als ein wesentliches Merkmal herausstellt. Bei Nietzsche ist es durch die Figur des Dionysos und die Anspielung auf die Tradition des Dithyrambus schon im Titel gegeben. Dem im Gedicht thematisierten Verhältnis des Ich zu etwas, das als göttlich begriffen ist, wenden wir uns im nächsten und letzten Abschnitt dieses Textes zu. Aber nochmals wird hier unterstrichen: spezifisch in „Letzter Wille“ fehlen die Anreden an göttliche oder als göttlich begriffene Instanzen.

\footnotetext{
${ }^{16}$ Wie D. Burdorf (2004: 306) pointiert, fällt die Verifizierung solcher stilistischen Eigenschaft schwerer aus.
} 
SERPA, D. - Untersuchungen der Dionysos-Dithyrambe "Letzter Wille"

Bevor wir zum nächsten Punkt übergehen, noch ein Wort über die Freien Rhythmen: generell bedient sich ihre beschreibende Analyse einer Analogie zu der antiken Metrik und ihren Versfüßen; Analogie, in der Hebungen und Senkungen mit den Längen und Kürzen der antiken Verse identifiziert sind. Das hat wiederum Wurzeln in theoretischen und dichterischen Versuchen, die deutsche Metrik der antiken anzugleichen. Für Kommerell (1956: 432f.) entstehen die Freien Rhythmen in der deutschen Literatur aus der Bemühung, die deutsche mit antiken Sprachen wetteifern zu lassen, aemulari (Paradebeispiel sind hier die Versuche Klopstocks, antike Odenverse und den Hexameter nachzuahmen). Die Analogie zwischen Hebungen und Senkungen einerseits und Längen und Kürzen andererseits kennt aber auch ihre Grenzen und viele Unstimmigkeiten; sie hat eine konventionelle Seite, über die die metrische Beschreibung des Gedichts hier nicht hinwegtäuschen soll und will (einen Überblick über die verschiedenen „Verssysteme“ und ihre [praktisch bis zu einem gewissen Grad unüberwindliche] Differenzen bietet KAYSER 1964 [1948]: 82-86): D. h., wenn hier von Versfüßen nach dem Modell der antiken Metrik die Rede ist, sind bestimmte Sequenzen von Hebungen und Senkungen gemeint.

R. Bunia (2014: 48) schreibt über das deduktive Versprogramm, dem die Freien Rhythmen zuzuordnen sind, dass ,der Rezipient [...] keine Formel vor der Lektüre im Hinterkopf [hat]". Prinzipiell hat jede Zeile in diesem Versprogramm eine individuelle abstrakt metrische Gestalt. Und manchmal bieten sich verschiedene Alternativen zur Analyse und Beschreibung eines solchen Verses an, was nicht nur mit der Entscheidung zu tun hat, ob eine Silbe eine Hebung oder Senkung darstellt, sondern auch damit, wie eine Sequenz von Hebungen und Senkungen zu gliedern sei.

In der kurzen Analyse der Verse von „Letzter Wille“ zu Beginn dieses Abschnittes konnte vermutlich gezeigt werden, dass die Freien Rhythmen dieses Gedichts, seine Sequenzen von Hebungen und Senkungen u. a. an einem kompositorischen Prinzip der Widerspiegelung teilhaben, die in den DD vielfach wirksam ist. Formen der Reflexion, der ein ,vernichtender' Zug zugeschrieben ist, spielen im Zyklus eine wichtige Rolle. Das lässt sich fast paradigmatisch am Gedicht „Zwischen Raubvögeln“ beobachten: Die Trennungen und Verdopplungen des Ich durch einen so weit gehenden Prozess der Reflexion (KSA 6: 390, VV. 41-52) bewirken, dass das Ich auf der Suche nach Selbsterkenntnis sich selbst verkennt und Objekt einer Verfolgung wird. 
SERPA, D. - Untersuchungen der Dionysos-Dithyrambe "Letzter Wille"

\section{Die Figur des, Freundes'}

„Letzter Wille“ kreist um das Thema des als vorbildhaft betrachteten Todes des Freundes, präsentiert da aber disparate Bilder. Wie Groddeck (1991b: 98) schreibt, ist das Gedicht nicht nur wegen seines esoterischen Charakters düster. Trotzdem ist es beim Heranziehen des Werks des Autors nicht so abwegig, in der Figur des Freundes Dionysos zu identifizieren - oder, vielleicht genauer ausgedrückt, eine dionysische Figur. Darin sind zumindest einige seiner Züge zu erkennen. Der ,Blitz' (KSA 6: 388, V. 3) erscheint im Zusammenhang mit diesem Gott auch in der „Klage der Ariadne“ (KSA 6: 400, V. 66; 401, V. 106) - vermutlich eine Bearbeitung des Motivs seiner Geburt, wenn Semele ihn

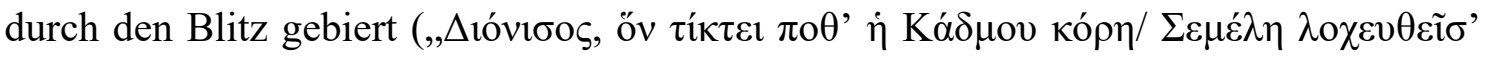
$\dot{\alpha} \sigma \tau \rho \alpha \pi \eta \varphi o ́ \rho \omega ~ \pi v \rho i ́$ “, am Eingang der Bakchen, VV. 2-3, von Euripides [2003: 129]). In den DD meint er auch den Geistesblitz (z. B. KSA 6: 402, V. 10). Sicherlich ist die Bezeichnung „ein Tänzer“ (KSA 6: 388, V. 6) die Charakterisierung, die am meisten Dionysos aufruft (emphatisch bringt der letzte Chorgesang der Antigone den Tanz in Beziehung zu dem Gott, SOPHOKLES 2009: 86-88, VV. 1115-1154). Selbst „mutwillig“ (KSA 6: 388, V. 5) rückt ihn in die Sphäre des Willens, der in der Verflochtenheit von Referenzen in der Geburt der Tragödie viel für Dionysos steht (NK 1/1 87; KSA 1: 4951). ${ }^{17}$

Die dionysische Figur des Gedichts zeigt Verwandtschaft auch mit einer anderen wichtigen Figur im Werk Nietzsches, deren Annährung an Dionysos in Also sprach Zarathustra am Ende doch vermieden wird (s. dazu GRODDECK 2017: 318-320), Zarathustra nämlich. Dieser wird schon in der „Vorrede“ des Buches, dessen Titel seinen Namen trägt, als ein Tänzer beschrieben (KSA 4: 12; im 4. Teil, KSA 4: 366). Leicht und schwer sind Attribute, die ihn in Ecce Homo (KSA 6: 345) beschreiben. Da bekommt Zarathustra auch die Bedeutung eines Schicksals (KSA 6: 343). Nach einem anderen Ausdruck dieses Buches (Ecce Homo) verkörpert Zarathustra „den Begriff des Dionysos“ (KSA 6: 344-345), also etwas, womit sich der Autor fast sein Leben lang fundamental auseinandergesetzt hat. Und in Ecce Homo schreibt Nietzsche noch: „Für eine dionysische Aufgabe gehört die Härte des Hammers, die Lust selbst am Vernichten in entscheidender Weise zu den Vorbedingungen“ (KSA 6: 349).

\footnotetext{
${ }^{17}$ Für weitere Hinweise auf Dionysos und das Dionysische bei Nietzsche in der Charakterisierung des „Freundes“, s. auch NK 6/2: 673. 
SERPA, D. - Untersuchungen der Dionysos-Dithyrambe "Letzter Wille"

Das Bild des Hammers - als dessen Rede wird eine Passage von Zarathustra vorgestellt (KSA 6: 161) - wird häufig im späten Werk des Autors verwendet. Es verbindet sich mit dem Projekt der Umwertung der Werte z. B. in dem alternativen Titel von Götzen-Dämmerung (KSA 6: 55). Das semantische Feld ihres Vorwortes, welches die „Aufgabe“ der „Umwerthung aller Werthe“ erwähnt, zeigt Analogien zu dem von „Letzter Wille“, zumal da von Schicksal die Rede und die Schrift als Kriegserklärung bezeichnet ist (KSA 6: 57-58). Dionysos steht in Beziehung dazu, indem er die „Lust des Werdens" meint, „die auch noch die Lust am Vernichten in sich schliesst" (KSA 6: 160). Aber die dionysische Bewegung greift auch über das Objekt hinaus und wendet zu sich zurück. Sie hat einen tragischen, selbstvernichtenden Zug, ${ }^{18}$ auf den wir unten kurz eingehen.

Beim späten Nietzsche wird Zarathustra Dionysos nicht nur angenähert, sondern auch auf gewisse Weise mit ihm gleichgesetzt:

man höre, wie Zarathustra vor Sonnenaufgang (III, 18) mit sich redet: ein solches smaragdenes Glück, eine solche göttliche Zärtlichkeit hatte noch keine Zunge vor mir. Auch die tiefste Schwermuth eines solchen Dionysos wird noch Dithyrambus (KSA 6: 345; meine Hervorhebung).

Diese Äußerungen über den Dithyrambus zeugen von eigenartigen Vorstellungen, die Nietzsche, Kenner der Tradition, mit dieser Poesie verbindet. Groddeck (1991b: XVIII) sieht die Bestimmung des Dithyrambus in den DD hauptsächlich in seiner Beziehung zu Dionysos, der aber wiederum vor allem eine Referenz im eigenem Werk Nietzsches ist (id. ibid.: bes. XIII-XVIII). I. Bach und H. Galle (1989: 311) schreiben: „Dithyrambus ist also für ihn [Nietzsche] keineswegs eine formale Bestimmung, die Auskunft über einen bestimmten Gedichttypus gibt; er ist vielmehr Ausdruck des Dionysischen, das auch noch über Schwermut, über alle Abgründe hinweg zur Bejahung des Lebens findet““. Die Charakterisierung der Gedichte des Zyklus der DD als „Ausdruck des Dionysischen“ (BACH; GALLE 1989: 311) lässt sich auf zwei Weisen geltend machen. Dionysos, das Dionysische ist ein Thema der Dithyramben, Objekt der Darstellung. Aber es gibt auch die Bedeutung, dass sich, wenn nicht nur das Dionysische, Dionysos selber

\footnotetext{
${ }^{18}$ In dem Gedicht „Letzter Wille“ finden wir die zwei Elemente, die die Tragödie für den frühen Nietzsche (in der Geburt der Tragödie) ausmachen: das des Sehens, des „principii individuationis“ (KSA I: 28), das da also für Erkenntnis und die Selbsterkenntnis steht (KSA I: 40), und dessen Zerreißung. Nietzsche behauptet (KSA I: 63; 70ff.), dass der tragische Held der attischen Tragödie („,bis auf Euripides“, S. 71) eine Maske Dionysos' sei, dessen Vernichtung auch Sieg und insofern Durchsetzung seines Prinzips bedeutete.
} 
SERPA, D. - Untersuchungen der Dionysos-Dithyrambe "Letzter Wille"

in den Dithyramben artikuliert: in der „Klage der Ariadne“ erscheint er und spricht (KSA 6: 401: 106-111).

Wegen des Charakters des Gedichts ist es schwierig (oder es wäre zu reduktionistisch), den „Freund“ (KSA 6: 388, v. 3) auf eine bestimmte bekannte Figur festzulegen. Der Vergleich geht nicht restlos auf. Vielmehr handelt es sich um ein Bündel von Eigenschaften, die abstrakt verschiedene Aspekte von Dionysos ansprechen und dabei Attribute Apollons mit einbezieht, wie die Funktion, die Möglichkeit des Sehens (KSA 6: 388, VV. 2-4). Diese Annährung überrascht nicht, insofern Übergänge, Vermittlungen zwischen diesen beiden Gestalten, besonders in der letzten Phase des Autors, angedeutet sind. Generell gesehen, scheint der „Freund“ im Gedicht vor allem die Rolle eines alter ego zu haben. Da mag Nietzsche das weite Feld von Bedeutungen dieses Ausdruckes und des Wortes alter vorgeschwebt haben (zu alter ego, s. auch den Eintrag alter in Lewis \& Short: ${ }^{19}$ „, a second self“, in Bezug auf sehr nahe Freunde). Im Bereich des Umgangs mit dem, was traditionell dem Göttlichen angehört, ist noch eine Figur zu berücksichtigen, die - wie hier angenommen wird - dem „Freund“ auch Züge verleiht und dementsprechend identifikatorischen Charakter (wenn auch polemisch) für das Ich hat: Christus. Dies kann auf den ersten Blick überraschen und sogar unangemessen wirken, werden doch Christus und Dionysos sehr prononciert und explizit beim späten Nietzsche zu einem antithetischen Paar (sehr bekannt sind die letzten Wörter von Ecce homo geworden: „Dionysos gegen den Gekreuzigten“, KSA 6: 374). Dass Christus dennoch zum Selbstbildnis des Ich in Beziehung gesetzt wird, tritt zum Beispiel dadurch zutage, dass Nietzsche den Ausdruck, mit dem in der Bibel auf Jesus hingewiesen wird, ecce homo (Io. 19, 5), in Bezug auf sich selbst gebraucht, indem er ihn als Titel des Buches wählt, in dem er sich vor- und rückblickend seinem Schaffen und Leben zuwendet. $^{20}$

Die Annäherung beider Gestalten, Christus und Dionysos, ist in der Dichtung, besonders der deutschsprachigen, nicht fremd. Kulturgeschichtlich wird die Zeit, die Welt in ein vor und nach Christus geteilt. Er gilt als Achse der Zeit, Ende (ein ,vernichtender Zug von ihm) und Anfang einer Ära. In „Letzter Wille“ deutet sich die Gestalt Christus darin an, dass er „sterbend siegte“ (KSA 6: 387, v. 12). Dazu passt in einem anderen

\footnotetext{
19 www.perseus.tufts.edu/hopper/morph?l=alter\&la=la\#lexicon.

${ }^{20}$ Johann Figl (2002: 152) bezieht sich auf eine Notiz Nietzsches vom Herbst 1881, die Annäherungen zwischen ihm und Christus nahelegt: „Auch im Hinblick auf Jesus sagt Nietzsche: ,In ihm lebe ich auch schon und in manchen Dingen kommt in mir erst reif ans Tagelicht, was embryonisch ein paar Jahrtausend brauchte“".
} 
SERPA, D. - Untersuchungen der Dionysos-Dithyrambe "Letzter Wille"

Rahmen die Idee, dass Dionysos selbst ein Opfer ist, der in der Tragödie zur Schau gestellt wird, aber gerade dadurch sich, sein Prinzip, durchsetzt (KSA I: 63; 70ff.). Freilich ist im Gedicht „Letzter Wille“ nichts von Auferstehungs- oder Jenseitsvorstellungen zu erkennen. Gerade in dieser Hinsicht, in dieser Umdeutung eines traditionellen, christlich religiösen Verständnisses des ,Sieges' Christi (V. 12), die dann eine Idee der Bejahung (,jauchzend“, V. 11) des Lebens in allen seiner Aspekte, besonders des Leidens und Todes suggeriert, wird seine Gestalt in eine Sphäre des Dionysischen hineingerissen. Angenähert werden diese beiden Gestalten mittels der Ausblendung, Beseitigung einer christlich erlösenden Perspektive. Diese Beseitigung ermöglicht, einen Unterschied zwischen der christlichen und der dionysischen bzw. tragischen Perspektive zu verwischen, den Nietzsche in einem Nachlassfragment vom Frühjahr 1888 so definiert: „Im ersten Falle [im christlichen Sinn] soll es [das Leiden] der Weg sein zu einem seligen Sein, im letzteren gilt das Sein als selig genug, um ein Ungeheures von Leid noch zu rechtfertigen." (KSA 13: 266) Dieser letzte Fall charakterisiert für ihn den tragischen Sinn, wofür Dionysos im zitierten Nachlassfragment steht. Wenn die Hoffnung hier noch gilt, die Nietzsche verschiedentlich durch sein Werk hindurch geäußert hat, dass der tragische Sinn und damit das Dionysische wiedergeboren werde $^{21}$, dann steht wahrscheinlich noch diese Umwertung vor Augen. „Letzter Wille“ bedeutet im Zusammenhang keine Auslöschung oder gar einfach Erfüllung, sondern eine Kontinuität, Fortsetzung des Willens.

\section{Rück- und Ausblicke}

„Letzter Wille“ stellt ein besonderes Gebilde für die DD dar. Das Gedicht ist merklich kürzer als die anderen des Zyklus; an ihm fehlt die Häufung von Elementen wie Anreden, Inversionen des syntaktischen Gefüges, Bildung von Komposita, die die DD insgesamt prägen. Gemeinsam hat es mit ihnen die Freien Rhythmen (mag auch seine Teilnahme am Zyklus auf diese metrische Klassifizierung wirken). „Anleihen bei antiken Versformen“ (SCHULZ; WERNER 2007: 253) müssen hier generell anders als in der freirhythmischen Dichtung des 18 Jh.s aufgefasst werden, aber Rekurse auf die deutschsprachige Poesie dieser Zeit können unterstellt werden. Vor allem wurden in der

\footnotetext{
${ }^{21}$ Ein früheres Beispiel dafür befindet sich in der vierten „Unzeitgemäßen Betrachtung: Wagner in Bayreuth" (KSA 1: 453).
} 
SERPA, D. - Untersuchungen der Dionysos-Dithyrambe "Letzter Wille"

Analyse Goethes „Lied der Parzen“ und Hölderlins „Hyperions Schicksalslied“ herangezogen, in denen eine Resonanz des Versfußes Amphibrachys zu finden ist. Zudem behandeln die drei Gedichte ein gleiches Thema, das Schicksal. Aber in der Bearbeitung dieses Stoffs unterscheiden sich die genannten Gedichte Hölderlins und Goethes einerseits und „Letzter Wille“ andererseits in ihrer Darstellung des menschlichen Schicksals im Verhältnis zu einer göttlichen Sphäre.

Im Vergleich zu Klopstock und Hölderlin, die freirhythmische Gedichte geschrieben haben, findet sich in dem lyrischen Werk Nietzsches nicht jene intensive dichterische Beschäftigung mit antiken metrisch festen Formen der Poesie. Aus dieser Perspektive überrascht nicht, dass ein bestimmter Ausgangspunkt der Freien Rhythmen dieses Autors in der antiken metrischen Dichtung nicht festzustellen war. Außerdem, wie C. Wagenknecht (1993: 92) schildert, ist die „Bindung an klassische Versmaße“ (s. 100) manchmal sehr diffus sowohl in der freirhythmischen Dichtung des 18. als auch - und vor allem - in der des 20. Jh.s.

Innerhalb des lyrischen Werkes Nietzsches konnten wir eine Erweiterung des metrischen Gefüges beobachten. In „Letzter Wille“ nimmt die Konfiguration von Hebungen und Senkungen an einem Prozess der Spiegelung teil, der sich auch auf das Verhältnis des Ich zu dem „Freund“ ausdehnen lässt, wodurch das Ich sein Selbstverständnis modelliert. Diese Spiegelung, diese Identifikation gestaltet sich als ein agonistischer Prozess. Der „Freund“ charakterisiert sich in einem Spannungsfeld von Figuren, die häufig im Werk des Autors auftauchen, hauptsächlich Zarathustra und Dionysos. Das Thema der Spiegelung wird in einem anderen Rahmen in der nächsten Dithyrambe des Zyklus (DD 4) weiter bearbeitet.

Was die Gattungsfrage angeht, treffen auf das Gedicht Bestimmungen des Dithyrambus bei Nietzsche zu. Ein Motiv dieser Poesie, das er eigens umarbeitet, ist, dass der Dithyrambus ,Ausdruck des Dionysischen“ (BACH; Galle 1989: 311) ist. Das bedeutet auch, Dionysos bzw. dionysische Attribute zu gestalten, zu exponieren, erscheinen zu lassen, und schließt noch ein, dass das den „Begriff des Dionysos“ (KSA 6: 345) bei Nietzsche selbst meint, der übrigens sehr weit gefasst ist und sich durch verschiedene Begriffe und Schriften des Autors ausbreitet und verzweigt. Im Fall von „Letzter Wille“ waren Anspielungen darauf im Kontext des Werks des Autors zu finden, das dann für die in diesem Text vorgeschlagene Deutung eine Rolle gespielt hat. Obwohl es sich in der Analyse des Gedichts gezeigt hat, dass der Begriff des Dithyrambus bei 
SERPA, D. - Untersuchungen der Dionysos-Dithyrambe "Letzter Wille"

Nietzsche im Allgemeinen über ein paar formale Eigenschaften hinaus geht, schließt das nicht aus, dass andere Gedichte des Zyklus Sprachgebärden der Hymne und damit spezifischere, vielleicht traditionellere Charakteristiken der Gattung aufweisen können. Aber das ist in diesem Sinn noch zu prüfen.

\section{Literaturverzeichnis}

Primärliteratur

BIBLIA Sacra Vulgata. 5. ed. Ed. Roger Gryson. Stuttgart; Barueri: Deutsche Bibelgesellschaft; Gráfica da Bíblia, 2007.

EURIPIDES. As bacantes. Übers. u. Einf. Trajano Vieira. São Paulo: Perspectiva, 2003.

GOETHE, Johann Wolfgang. Dichtung und Wahrheit. Frankfurt am Main; Leipzig: Insel Verlag, 1975.

GoETHE, Johann Wolfgang. Fausto: uma tragédia - Segunda parte. Übers. Jenny K. Segall. São Paulo: Ed. 34, 2007.

GoETHE, Johann Wolfgang. Klassische Dramen. Hrsg. D. Borchmeyer. Frankfurt am Main: Deutscher Klassiker Verlag, 2008.

HERDER, Johann Gottfried. „Pindar und der Dithyrambensänger“. In: HERDER, Johann Gottfried. Ueber die neuere Deutsche Litteratur. Zwote Sammlung von Fragmenten. Eine Beilage zu den Briefen, die neueste Litteratur betreffend. Riga: Hartknoch, 1767, 298-338. Erreichbar unter: https://www.uni-due.de/lyriktheorie/texte/1767_2herder.html\#text. Letzter Zugang: 31/08/2021

HÖLDERLIN, Friedrich. Sämtliche Werke und Briefe. Ed. Jochen Schmidt. 3 Bd. Frankfurt am Main: Deutscher Klassiker Verlag, 1992-1994. [zitiert als SW]

HoraCE. Odes and Epodes. Transl. by C. E. Bennett. Cambridge; Massachusetts; London: Harvard University Press, 1995.

KLOPSTOCK, Friedrich Gottlieb. Oden. Auswahl und Nachwort von Karl Ludwig Schneider. Stuttgart: Reclam, 2006.

NIETZSCHE, Friedrich. Digital critical edition of the complete works and letters, based on the critical text by G. Colli and M. Montinari. Ed. by Paolo D'Iorio. Berlin; New York: de Gruyter 1967. [zitiert als eKGWB]

NIETZSCHE, Friedrich. Sämtliche Werke. Kritische Studienausgabe in 15 Bänden. 10. ed. Ed. Giorgio Colli u. Mazzino Montinari. München: Deutscher Taschenbuchverlag, 2015. [zitiert als KSA]

SóFloClES. Antígone. Übers. u. Einf. Trajano Vieira. São Paulo: Perspectiva, 2009.

Sekundärliteratur

BACH, Inka; GALLE, Helmut. Deutsche Psalmendichtung vom 16. bis zum 20. Jahrhundert: Untersuchungen zur Geschichte einer lyrischen Gattung. Berlin; New York: de Gruyter, 1989.

Bowers, K.; Chisholm, D. H. "Freie Rhythmen". In: Greene, R. et al. (Hrsg.). The Princeton Encyclopedia of Poetry and Poetics. 4. ed. New Jersey: Princeton University Press, 2012, 525 .

Pandaemonium, São Paulo, v. 25, n. 45, jan.-abr. 2022, p. 136-156 
SERPA, D. - Untersuchungen der Dionysos-Dithyrambe "Letzter Wille"

BREMER, Jan M.; FurLeY, William D. “Introduction”. In: BREMER, Jan M.; FurLEY, William D. Greek Hymns. Vol. 1. Tübingen: Mohr Siebeck, 2001, 1-64.

BRUSOTTI, Marco. „Dionysos-Dithyramben (1888/1889)“. In: OTTMANN, H. (hrsg.). NietzscheHandbuch. Stuttgart; Weimar: Metzler, 2011, 136-137.

BUNIA, Remigius. Metrik und Kulturpolitik. Berlin: Ripperger \& Kremers, 2014.

BURDORF, Dieter. „Gibt es eine Geschichte der deutschen Hymne?“. Zeitschrift für deutsche Germanistik, 14, 298-310, 2004.

BURDORF, Dieter. Einführung in die Gedichtanalyse. 3. ed. Stuttgart: Metzler Verlag, 2015.

FIGL, Johann. „,Dionysos und der Gekreuzigte': Nietzsches Identifikation und Konfrontation mit zentralen religiösen ,Figuren““. Nietzscheforschung, v. 9, 147-162, 2002.

GRODDECK, Wolfram. „'Ein andres Wort für Musik'. Zu Friedrich Nietzsches Venedig-Gedicht“. In: HARTung, H. (Hrsg.) Gedichte und Interpretationen. Band 5: Vom Naturalismus bis zur Mitte des 20. Jahrhunderts. Stuttgart: Reclam, 2011, 20-32.

GRODDECK, Wolfram. Friedrich Nietzsche - Dionysos-Dithyramben. Bd. 1. Berlin; New York: De Gruyter, 1991a.

GRODDECK, Wolfram. Friedrich Nietzsche - Dionysos-Dithyramben. Bd. 2. Berlin; New York: De Gruyter, 1991b.

GRODDECK, Wolfram."Die Wahrheit im Dithyrambus. Zu Nietzsches 'Dionysos-Dithyramben'”. In: BENNE, Christian; ZITTEL, Claus (Hrsg.). Nietzsche und die Lyrik. Stuttgart: J. B. Metzler, 2017, 317-330.

HIEBEL, Hans H. Das Spektrum der modernen Poesie (Teil I 1900-1945). Würzburg: Königshausen \& Neumann, 2005.

KAYSER, Wolfgang. Das sprachliche Kunstwerk (1948). 10. ed. Bern; München: Francke, 1964.

KOMMERELL, Max. Gedanken über Gedichte. Frankfurt am Main: Klostermann, 1956.

LEWIS \& Short Latin Dictionary. Perseus Digital Library Tufts University. http://www.perseus.tufts.edu/hopper/resolveform?redirect=true\&lang=Latin.

MENNINGHAUS, Winfried. „Zwischen Überwältigung und Widerstand. Macht und Gewalt in Longins und Kants Theorien des Erhabenen."Poetica, v. 23, n. 1/2, 1-19, 1991.

MoennighofF, Burkhard. „Freie Verse“. In: Burdorf, D.; FASBEnder, C.; MoEnNighofF, B. (Hrsg.) Metzler Lexikon Literatur. Stuttgart; Weimar: Metzler, 2007, 253-254.

MoEnNighofF, Burkhard; Schulz, Georg-Michael. "Freie Rhythmen". In: BurdoRf, D.; FASBender, C.; Moennighoff, B. (Hrsg.) Metzler Lexikon Literatur. Stuttgart; Weimar: Metzler, 2007, 253.

Oestersandfort, Christian. "Hymne". In: Burdorf, D.; FAsbender, C.; Moennighoff, B. (hrsg.). Metzler Lexikon Literatur. Stuttgart; Weimar: Metzler, 2007, 333-335.

SCHMIDT, Jochen. Kommentar zu Nietzsches die Geburt der Tragödie. Band 1/ 1. Berlin; Boston: De Gruyter, 2012 [zitiert als NK 1/1].

SERPA, Danilo. "Seguindo lei firme, como outrora, gerado do caos sagrado, sente-se de novo o entusiasmo": a representação do poeta e do seu fazer no hino alemão de Klopstock, Goethe e Hölderlin. 216f. Dissertação (Mestrado em Língua e Literatura Alemã) Faculdade de Filosofia, Letras e Ciências Humanas, Universidade de São Paulo, São Paulo, 2014.

Sommer, Andreas Urs. Kommentar zu Nietzsches Der Antichrist, Ecce homo, DionysosDithyramben, Nietzsche contra Wagner. Band 6/2. Berlin; Boston: De Gruyter, 2013 [zitiert als NK 6/2].

Steinhagen, Harald. „Amphibrachys“. In: Burdorf, D.; FASBender, C.; Moennighoff, B. (hrsg.). Metzler Lexikon Literatur. Stuttgart; Weimar: Metzler, 2007, 19.

SzONDI, Peter. „Interpretationsprobleme (Hölderlin: Feiertagshymne, Friedensfeier)“. In: SzONDI, Peter. Einführung in die literarische Hermeneutik. Frankfurt am Main: Suhrkamp Verlag, 1975, 193-401.

Pandaemonium, São Paulo, v. 25, n. 45, jan.-abr. 2022, p. 136-156 
SERPA, D. - Untersuchungen der Dionysos-Dithyrambe "Letzter Wille"

VÖHLER, Martin. Pindarrezeptionen. Sechs Studien zum Wandel des Pindarverständnisses von Erasmus bis Herder. Heidelberg: Universitätsverlag Winter, 2005.

WAGENKNECHT, Christian. Deutsche Metrik: Eine historische Einführung. 3. ed. München: Beck, 1993.

WERLE, Dirk. "Klopstocks Probleme: der doppelte Anfang der deutschen Hymne". Zeitschrift für Deutsche Philologie, Band 131, Heft 4, 481-511, 2012.

ZIEMANN, Rüdiger. „Gedichte“. In: OtTMANN, H. (Hrsg.). Nietzsche-Handbuch. Stuttgart; Weimar: Metzler, 2011, 150-156.

Recebido em 21 de dezembro de 2020 Aceito em 11 de maio de 2021 